\title{
ROLE OF DYNAMIC CONTRAST-ENHANCED AND DIFFUSION- WEIGHTED MRI IN EVALUATION OF RESPONSE OF HEPATOCELLULAR CARCINOMA AFTER CHEMOEMBOLIZATION.
}

\author{
Mounir S. Guirguis ${ }^{1}$, Sherine G.Moftah ${ }^{1}$, Yasser I. AbdElkhalek ${ }^{1}$, Wael F. \\ Elshawaf ${ }^{2}$, Ahmed M. Mohammed ${ }^{2}$
}

1`Department of Radio-diagnosis, Faculty of Medicine, Ain Shams University,

2-Department of Radio-diagnosis, Medical Military Academy, Cairo, Egypt.

Corresponding :

Ahmed Mustafa Mohammed

Mobile: Tel. 01091205400

E mail:

ahmed.mustafa98989@gmail.com

Received: $13 / 11 / 2019$

Accepted: 11/12/2019

\begin{abstract}
:
Background: Hepatocellular carcinoma (HCC) accounted as the sixth most prevalent cancer worldwide and the third most common cancer leading to death. However unfortunately only a minority HCC patients are surgical candidates at the time of diagnosis, Transcatheter arterial chemoembolization (TACE) is one of the most commonly used intra-arterial therapies to treat unresectable HCC, Assessing early response to therapy using objective criteria is paramount for clinical care.
\end{abstract}

Aim of the Work: To emphasize the role of dynamic contrast-enhanced and diffusion-weighted MRI in the assessment of response to treatment of hepatocellular carcinoma after transcatheter arterial chemoembolization (TACE).

Patients and Methods Thirty-eight patients who were proven as HCC patients radiologically with AFP correlationwere enrolled in this study, fulfilled the inclusion criteria. They scheduled to undergo dynamic MRI with DWI within 90 days after one or more treatments of TACE. In case of absent evidence of residual, follow up was done within 90 days after the first MRI.

Results no statistically significant difference between group according to demographic data, and lesion size, while there is statistically significant relation between groups according to signal intensity $(P<0.001)$, dynamic enhancement $(P<0.001)$, DWIs $(P=0.036)$ and $A D C$ value $(P<0.001)$.

Conclusions dynamic MRI is still superior than Diffusion-weighted MR imaging with high specificity and remain the standard follow up technique in well cooperative patient yet DWI adding more to the overall sensitivity when combined with it and may help in condition of difficulty breath holding.

Keywords: Diffusion Weight Magnetic resonance image, Dynamic enhancement Magnetic resonance image

\section{INTRODUCTION}

Hepatocellular carcinoma counted as the sixth most prevalent cancer worldwide and the third most common cancer leading to death. However, unfortunately, only a minority of HCC patients are surgical candidates at the time of diagnosis with Transcatheter arterial chemoembolization (TACE) is one of the most commonly used intra-arterial therapies to treat unresectable $\mathrm{HCC}^{(1)}$. The evaluation of tumour response of hepatic malignancies after intra-arterial therapies is of major clinical interest, However, imaging techniques and imaging response criteria have been limited in giving clinically satisfactory information about the extent of tumour necrosis ${ }^{(2)}$.

Unenhanced CT is a superior modality for evaluation of the pattern of acculmulation of the chemoembolization mixture into the targeted lesions yet its beam 
hardening artefacts produced by the high attenuation of iodized oil make obscure the contrast enhancement of the residual tumoral tissue while the signal intensity of MRI is not degraded by the presence of iodized oil; therefore, a residual viable tumour is better defined by $\mathrm{MRI}^{(3)}$.

Conventional Non-contrast T1- and T2weighted images provide information on morphological change, fluid content and fibrosis, and dynamic contrast-enhanced MRI can provide information on perfusion for assessment of the response to treatment ${ }^{(4)}$.

Diffusion can provide insight about water composition within a tumour and the degree of tumour viability. Viable tumour cells have intact membranes that restrict water diffusion, whereas necrotic tumours have increased water diffusion due to cell membrane distruption ${ }^{(5)}$ The apparent diffusion coefficient (ADC) calculated in diffusion-weighted MRI is a measure of the mobility of water in tissues. Viable tumours cells have an intact cell membrane that restricts the mobility of water molecules and results in a relatively low ADC. Conversely, cellular necrosis increases membrane permeability, allowing water molecules to move freely and causing a relative increase in $\mathrm{ADC}^{(3)}$

The World Health Organization (WHO) guidelines, incorporating bidimensional perpendicular measurements and the Response Evaluation Criteria in Solid Tumors (RECIST) incorporating unidimensional measurements, were intended to evaluate change in tumor size over months to years after systemic treatments without considering the changes in tumor vascularity or necrosis New criteria taking into account a decrease in enhancing tumour and tumour necrosis on CT and MRI have been proposed. The criteria proposed by the European Association for the Study of the Liver (EASL) are based on modified WHO bidimensional measurements to estimate tumour response while The introduced modified RECIST classification addresses many of the shortcomings of the EASL criteria by defining methods for newer image acquisitions, target selection, and target measurement by adapting many of the strengths of RECIST. Modified RECIST uses the single largest diameter of the viable tumour (defined as the component enhancing during the arterial phase) and is more practical for clinical use ${ }^{(6)}$.

\section{MATERIAL AND METHODS:}

\section{Study population}

Thirty-eight patients had undergone MR imaging over a period of 24 months (February 2016-February 2018), studies were performed at Maadi Military Hospital. All patients were subjected to full clinical assessment, revise of theirs laboratory including renal function tests and previous radiological investigation.

The patients' age ranged from 44 to 75 years (median 66.6) 35patients were males and 3 were females. All patients had liver cirrhosis related to chronic viral hepatitis.

All patients scheduled to undergo dynamic MRI with DWI within 90 days after one or more treatments of TACE. In case of absent evidence of residual, follow up was done within 90 days after the first MRI.

\section{Trans arterial chemoeembolization (TACE):}

Chemoembolization is performed percutaneously in the angiography suite, with the patient under conscious sedation. After infiltration of local anaesthetic, the Seldinger technique is used to gain access to the common femoral artery through femoral artery puncture, although the brachial artery may also be used ${ }^{(7)}$.

A 5-French vascular sheath is placed into the common femoral artery over a 
0.035-inch Guide-wire. Under fluoroscopic guidance, a 5-French glide Simmons-1 or Cobra catheters (Cordis, Miami, FL) is advanced into the aortic arch, formed, and then used to select the celiac axis ${ }^{(8)}$.

\section{Image Acquisition}

\section{Magnetic resonance imaging:}

The MRI was performed using a 3-T MRI scanner GE\{ Discovery 750$\}$ equipped with phased-array torso surface coil.

\section{Protocol of MRI:}

Precontrast T1 3D-SGE FS pre axial weighted image $(\mathrm{TR}=3.8 \mathrm{msec}, \mathrm{TE}=1.7$ msec, $\mathrm{FOV}=350 / 400 \mathrm{~mm}$, acquisition matrix $=160 / 256$ pixels, slice thickness $=3 \mathrm{~mm}$, with $1 \mathrm{~mm}$ gap). T2 SS-FSE axial weighted images its value $(\mathrm{TR} \geq 1500 \mathrm{msec}, \mathrm{TE}=70$ $85 \mathrm{msec}, \quad \mathrm{FOV}=350 / 400 \mathrm{~mm}$, acquisition matrix $=192 \times 256$ pixel, slice thickness $=6$ $8 \mathrm{~mm}$, with 1mm gap).

DWI was performed before the dynamic imaging using respiratory triggered fatsuppressed single-shot echoplanar sequence by applying three different $b$ factors of 20 , 500 , and $800 \mathrm{~s} / \mathrm{mm} 2$.Parallel imaging with generalized auto-calibrating partially parallel acquisition (GRAPPA) with an acceleration factor of two was applied to reduce the acquisition time. The other parameters were as follows: repetition time (TR) $\geq 5000 \mathrm{msec}$, echo time (TE) $66-70 \mathrm{msec}$, number of excitations $(\mathrm{NEX})=3$, matrix $112 / 256$ with a field of view as small as possible, slice thickness $6-7 \mathrm{~mm}$, slice gap $1-2 \mathrm{~mm}$, scan time 4-5 $\mathrm{min}$.

The dynamic study was done after the diffusion study to avoid the effect of contrast agents on $\mathrm{ADC}$ value. after bolus injection of $0.1 \mathrm{mmol} / \mathrm{kg}$ bodyweight of Gd-DTPA, flushed with $20 \mathrm{ml}$ of sterile saline solution from the antecubital vein. using T1 LAVA ( Liver Acquisition with Volume Acceleration ) technique performed in triphasic way; A dynamic series consisted of one pre-contrast series followed by four successive post- contrast series including early arterial, late arterial, and portal venous phase imaging with 18-21s intervals (17-20 s) for image acquisition according to liver size with breath-holding and one second for rebreathing for the start of each phase imaging followed by 5-min delayed phase imaging. All patients were imaged in end-expiration to limit the risk of image misregistration with Acquisition parameters were 3.34.5/1.4-1.9; flip angle, $10^{\circ}$; number of signals averaged, 1; parallel imaging factor, 1.8; matrix size, $172 \times 135$; field of view, 300-400 mm, slice thickness, $2-3 \mathrm{~mm}$.

\section{ADC measurement:-}

Pixel-based ADC maps were generated on the workstation. ADC was calculated with linear regression analysis of the function $\mathrm{S}=\mathrm{S} 0 \times \exp (-\mathrm{b} \times \mathrm{ADC})$, where $\mathrm{S}$ is the signal intensity after application of the diffusion gradient, and $\mathrm{S} 0$ is the signal intensity at $\mathrm{a} b$ value of $0 \mathrm{~s} / \mathrm{mm} 2$. The three $b$ values $(0,500$, and $800 \mathrm{~s} / \mathrm{mm} 2)$ were used for ADC calculation A region of interest was drawn over any sustaining hyperintensity areas on diffusion images, and if no high signal can be identified, the whole lesion was measured. The ADC was measured three times and the three measurements were averaged.

\section{MR images analysis and Interpretation}

\section{$M R$ images were analyzed for the following:}

- The morphological features of each lesion including size, border, signal intensity at $\mathrm{T} 1$ and $\mathrm{T} 2$ weighted images.

- The enhancement pattern at the dynamic contrast-enhanced imaging.

- Signal intensity in diffusion imaging with measurement of ADC values using a commercial windows workstation.

\section{Interpretation of the MR image:}

- The signal of the ablatedlesionat T1 and T2 was classified as high, low, heterogeneous. 
- Dynamic study interpretation:

a. Arterial enhancement and subsequent washout pattern were regarded as suggestive findings of the residual viable lesion.

b. The absence of enhancement in the arterial phase or progressive or persistent enhancement was detected on dynamic images was suggestive of the well ablated nonviable lesion.

\section{- Diffusion MRI:}

Signal intensity on diffusion images with ADC values and fusion images using commercially windows workstation (GE).The pattern of diffusion restriction was classified into- homogenous and nodular.

All DWI images using different $b$ values were sorted according to two grade scales:-

a. Nonviable lesions were considered when lost signal on diffusion images or mild sustained hyperintensity with bright ADC map (shine through effect) is noted.

b. Viable tumour portion was identified by sustained hyperintensity in the diffusion images compared with the signal drop of background parenchyma with increasing $b$ values combined with low ADC map.

$\mathrm{ADC}$ value measured three times and the three measurements were averaged.

\section{Statistical analysis}

- For statistical analysis Computer software package SPSS version 20 was used in the analysis. And a Chi-Square test was used to estimate differences in qualitative variables.

- A ROC (Receiver Operating Characteristic) curve was constructed and the area below the ROC curve was used to represent prediction precision.

- P-values less than 0.05 were considered as statistically significant.

\section{The standard of reference}

- It was difficult to obtain pathologic confirmation in patients who underwent chemoembolization because most of these patients do not undergo surgery. Few lesions were located at the hepatic dome and few cases were small in size. The aforementioned factors could cause selection bias leading to increased sensitivity of diffusion-weighted images since hepatic lesions close to the diaphragm pose a challenge to DW-MRI evaluation as they are more sensitive to motion and susceptibility artefacts.

- Viable HCC was finally diagnosed depending on thesustained iodized-oil accumulations in the hypervascular residual area on the hepatic arteriography at the following session of TACE.

- In the nonviable lesion the perilesional inflammatory, ischemic hepatic parenchyma changes abnormal signal intensity area should regress in size or totally disappears on MRI in 3 months follow-up. 

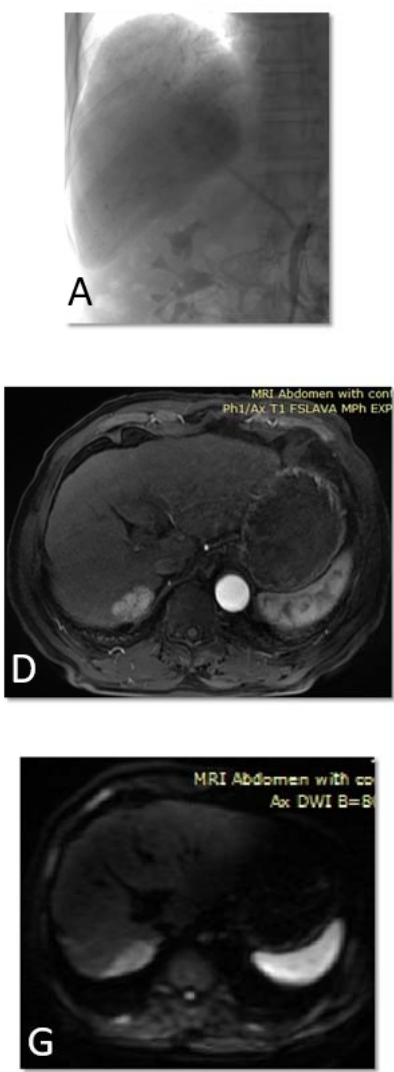
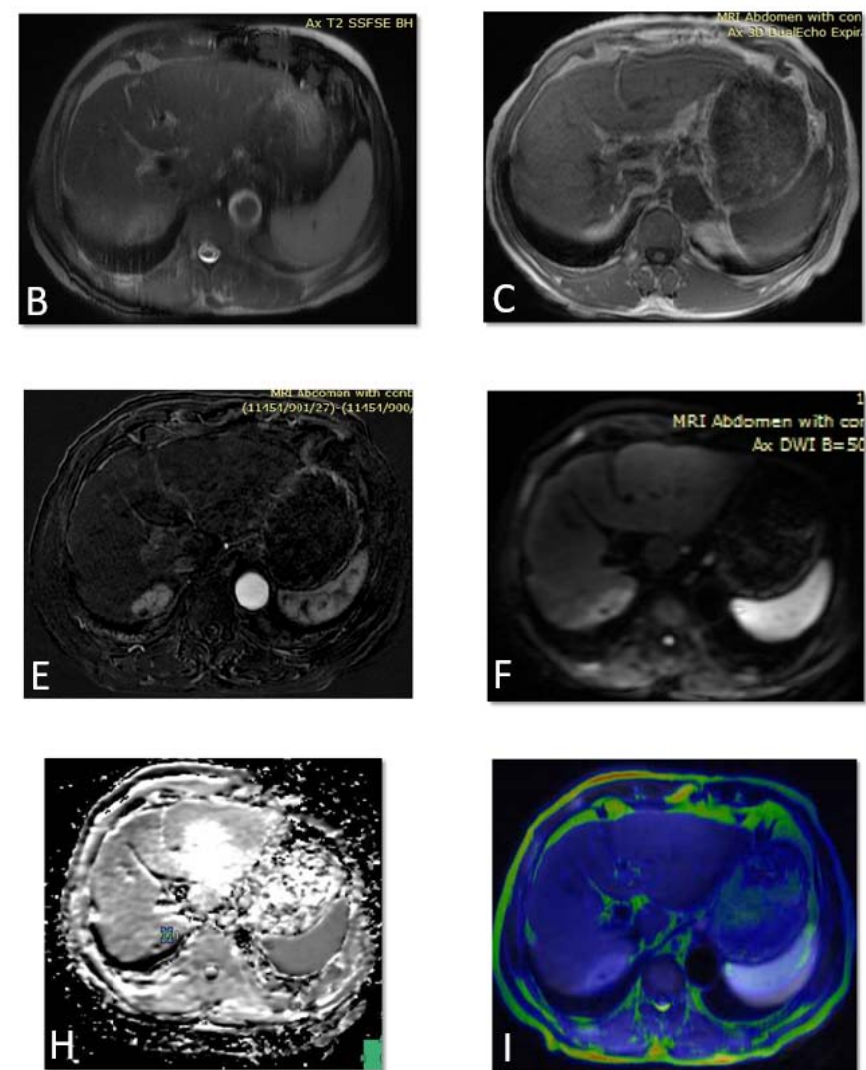
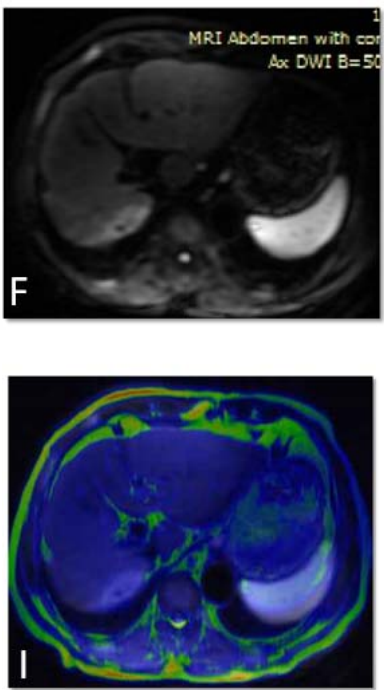
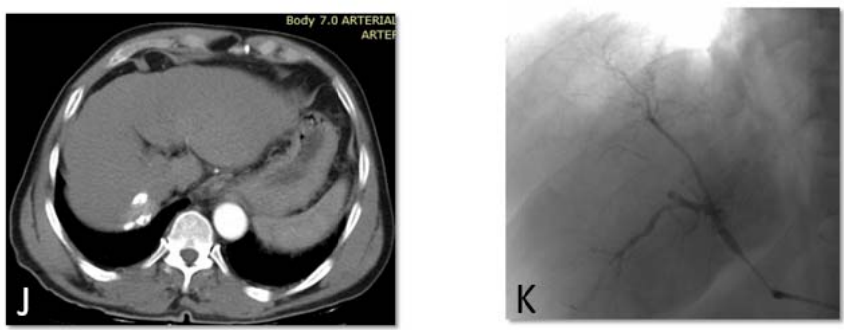

Fig. (1): Seventy-one-year-old male patient with hepatitis $\mathrm{C}$ with residual segment VI tumour lesion post-TACE.( A) Angiographic study prior to chemoembolization display tumoral blush,MRI after 2 month of chemo-embolization (B) Axial T2 SSFSE (repetition time/echo time, $1750.1 \mathrm{msec} / 82.1 \mathrm{msec}$ ) show high signal intensity of the lesion and (c) Axial 3D T1 WIs(repetition time/echo time, $3.2 \mathrm{msec} / 1.5 \mathrm{msec}$ ) revealed heterogeneous low signal intensity of segment VI lesion (D\&E) Early arterial phase T1 LAVA and its corresponding T1WI LAVA subtracted sequence showing avid arterial enhancement of the lesion with tiny nonenhancing area (F, G\& H) DWI(repetition time/echo time, $7571.4 \mathrm{msec} / 63.1 \mathrm{msec})$ of variable $(500$ and 800$) \mathrm{b}$ value with its generated ADC map showing solid restricted diffusion of the lesion with ADC value of $1.2 \pm 0.2 \times 10^{-3} \mathrm{~mm} 2 / \mathrm{sec}$, (I)Fusion image showing overlap between the high signal intensity of the lesion on T2 WIs and the restriction seen on diffusion images (b value 500) (J)Triphasic CT (arterial phase ) showing faint heterogenous accumulation of Lipidol with residual enhancing lesion (K) Angiograpgic image of the 2nd TACE session display residual mild blushing of the previously ablated lesion . 

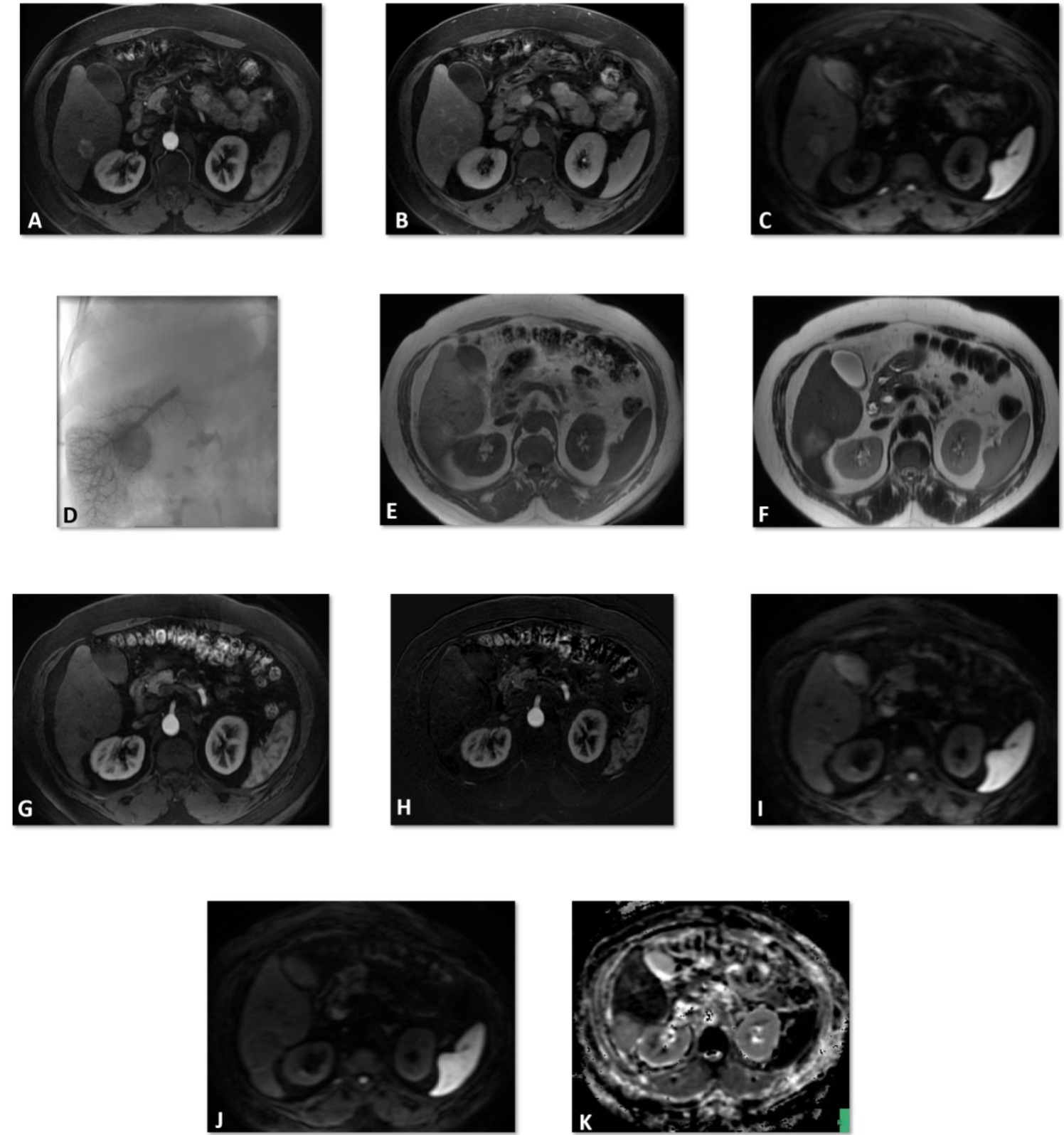

Fig. (2): Fifty four year old male patient with hepatitis C with segment VI focal lesion (A) Axial post Gd-DTPA T1 LAVA sequence revealed early arterial enhancement of the lesion, (B)delayed phase washout of the contrast with (C) restricted diffusion at b500 DWI sequence (D) Angiographic study prior to chemoembolization display tumoral blush, MRI after 1 month of chemo-embolization (E) Axial 3D T1 WIs(repetition time/echo time, $3.1 \mathrm{msec} / 1.4 \mathrm{msec}$ ) revealed high 'T1 signal intensity of chemoembolized lesion (F) Axial T2 SSFSE (repetition time/echo time, $1765.1 \mathrm{msec} / 80.4 \mathrm{~m} \mathrm{sec}$ ) shows high signal intensity of the lesion due to post chemoembolizeation tumoral hemorrhage $(\mathrm{G} \& \mathrm{H})$ Early arterial phase T1 LAVA and its corresponding T1WI LAVA subtracted sequence showing no arterial enhancement of the well ablated lesion. (I, J\&K) DWI(repetition time/echo time, $8351.3 \mathrm{msec} /$ $66.2 \mathrm{msec}$ ) of variable (500 and 800$) \mathrm{b}$ value with its generated ADC map showingfacilitated diffusion of the lesion with ADC value of $1.9 \pm 0.03 \times 10-3 \mathrm{~mm} 2 / \mathrm{sec}$.

\section{RESULTS}

Thirty-eight patients were included in our study. Their age ranged from 44 to 75 years with a mean of age (mean=61.66
$\mathrm{SD}=7.21$ ), they were 3 females and 35 males.

The patient categorized according to the LI-RADS algorism into LR- Treated, our involved cases classified as regard its post- 
therapeutic response into 24 patients LR- Nonviable. Treated viable and 14 patients LR-Treated

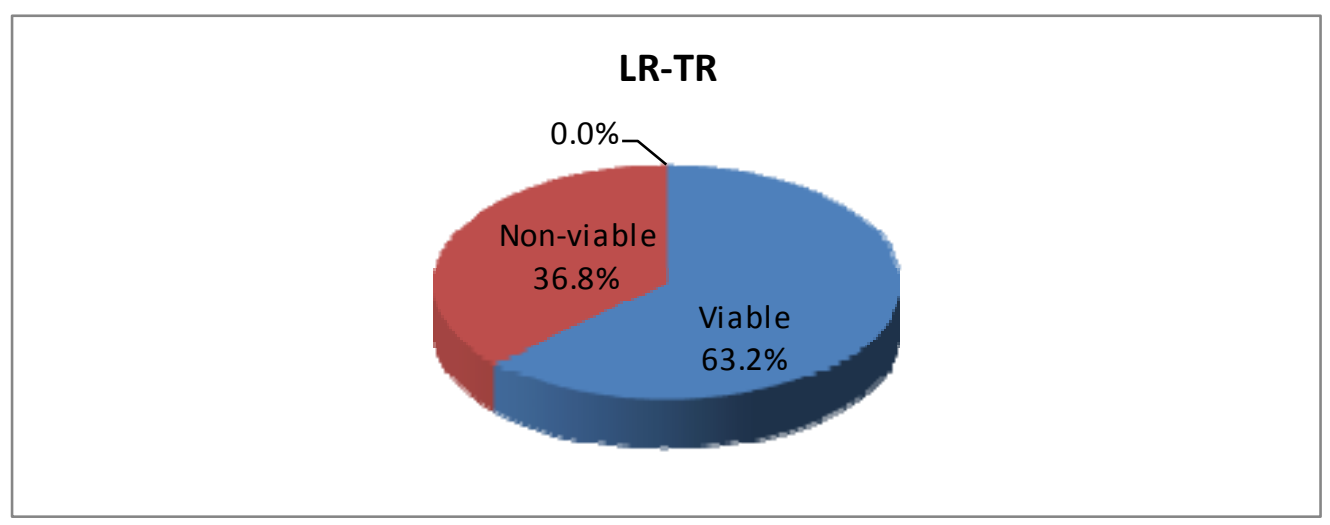

Diagram. (1): Bar chart LR-TR distribution of the study group.

Among the 24 LR-treated viable cases $(25.0 \%)$ showed heterogeneous and $(75 \%)$ show low T1 signal intensity and (25.0\%) showed heterogeneous and (75\%) show low T2 signal intensity, while in the 14 LRtreated nonviable cases $(14.3 \%)$ showed heterogeneous and $(85.7 \%)$ show low $\mathrm{T} 1$ signal intensity and (14.3\%) showed heterogeneous, $(21.4 \%)$ show high and (64.3\%)show low T2 signal intensity that makes the depend on conventional MRI sequence in assessment of HCC necrosis after TACE is confusing .

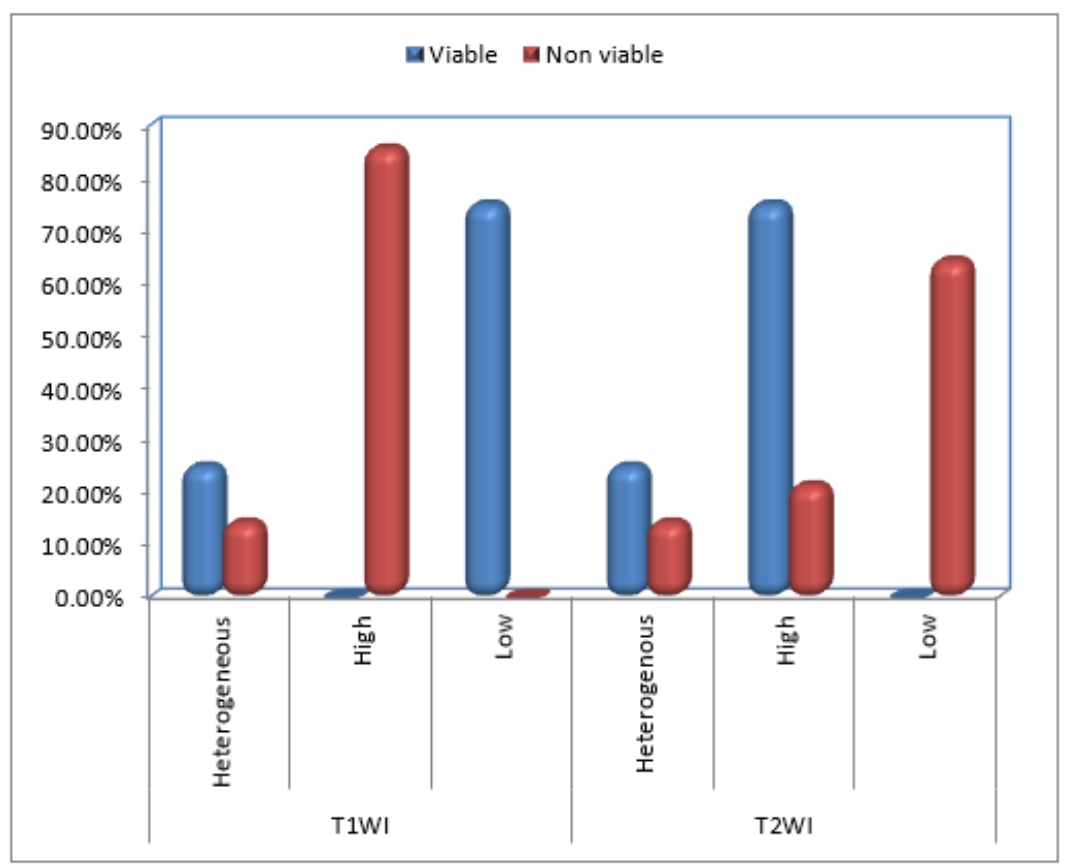

Diagram.(2): Bar chart between viable and non-viable according to signal intensity.

Dynamic MRI had a sensitivity of $95.8 \%$, a specificity of $92.9 \%$, a positive predictive value of $95,8 \%$, a negative predictive value of $92.9 \%$ and overall agreement of $91.4 \%$ compared to $70,8 \%$, $64.3 \%, \quad 77.3 \%, \quad 56,3 \%$ and $67,4 \%$ respectively of diffusion-weighted imaging. 
Table (1): Comparison between viable and non-viable according to dynamic enhancement and DWIs.

\begin{tabular}{|l|c|c|c|}
\hline & $\begin{array}{c}\text { Dynamic } \\
\text { enhancement }\end{array}$ & DWIs & Combined \\
\hline Sensitivity \% & $95.8 \%$ & $70.80 \%$ & $100 \%$ \\
\hline Specificity\% & $92.9 \%$ & $64.30 \%$ & $88.3 \%$ \\
\hline PPV\% & $95.8 \%$ & $77.30 \%$ & $90.2 \%$ \\
\hline NPV\% & $92.9 \%$ & $56.30 \%$ & $100 \%$ \\
\hline Accuracy\% & $94.7 \%$ & $68.40 \%$ & $95.1 \%$ \\
\hline Overall agreement & $91.4 \%$ & $67.40 \%$ & $94.7 \%$ \\
\hline
\end{tabular}

Receiver operating characteristics (ROC) curve was used to define the best cut off value of ADC value with $\leq 1.22$ has a sensitivity of $83.3 \%$, specificity $71.4 \%$, PPV $83.3 \%$, NPV $71.4 \%$ and accuracy $63.2 \%$.

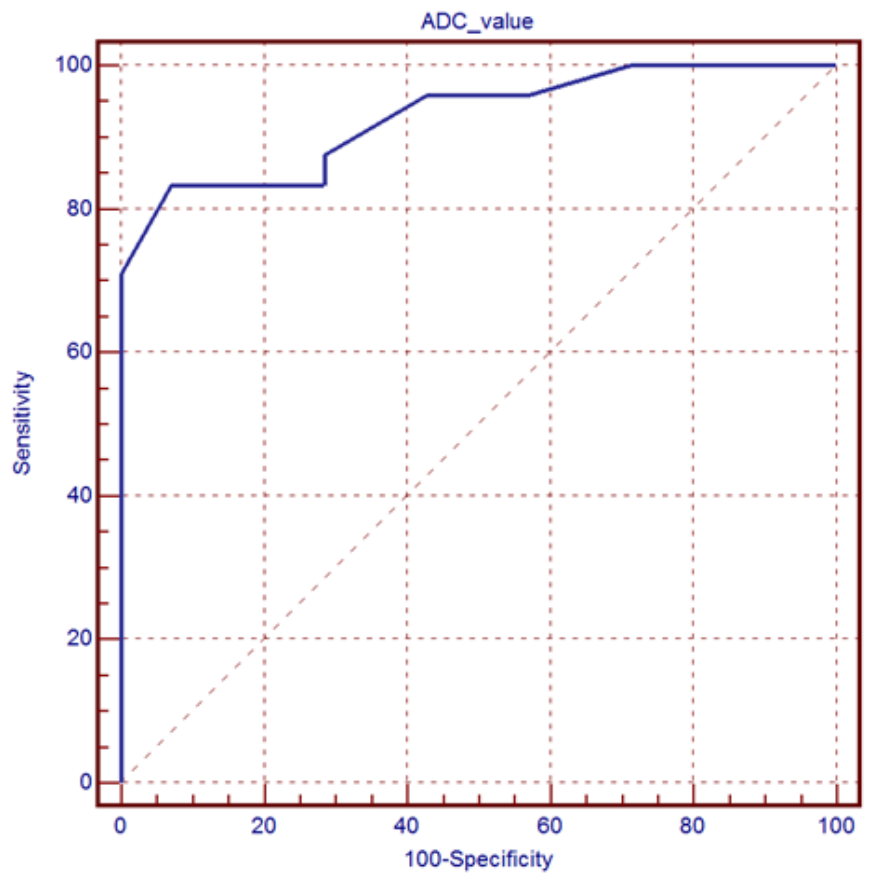

Diagram (3):Receiver operating characteristics (ROC), the diagnostic performance of viable and non-viable in discrimination of ADC value.

\section{DISCUSSION:}

As stated by Kim et al. ${ }^{(9)}$,that Multiphase dynamic CT is popularly used for evaluating the therapeutic effect of TACE because the degree of uptake and the distribution of the iodized oil within the tumour and the surrounding hepatic parenchyma can provide useful information on the degree of tumour necrosis However Lim et al. ${ }^{(3)}$. stated that it can be difficult to evaluate contrast enhancement in a tumour with partial retention of iodized oil on contrast-enhanced CT because of the beam hardening artefacts produced by the high attenuation of iodized oil.
As stated by Yaghmai et al. ${ }^{(6)}$ a central area of coagulative necrosis is often seen within the ablated tumour. This coagulative necrosis will result in a hyperintense signal on T1-weighted imaging and Braga et al. ${ }^{(10)}$ stated that Lesions that showed good response became low signal on T2-weighted images immediately after treatment, reflecting the devascularization of the tumor.

Özkavukcu et al. ${ }^{(11)}$ reported that In addition to residual tumour, T2 hyperintensity can represent haemorrhage, liquefactive necrosis, or inflammatory infiltrate in the current study, about $21.1 \%$ 
of lesions showed heterogeneous signal intensity that makes assessment of HCC necrosis after TACE depending on conventional spin-echo T1-weighted and T2weighted imaging characteristics after chemoembolization were highly variable.

Braga et al. ${ }^{(10)}$ stated that within 1 month after chemoembolization, Partial response shows enhancement on immediate postgadolinium images of residual tumour. However, Transient hyperemia manifested by thin, uniform enhancement of the treated zone is an expected finding after TACE and represents a transient physiologic response to embolizationof the hepatic parenchyma as mentioned by Yaghmai et al. ${ }^{(6)}$

In the current study, we had a sensitivity of dynamic MRI agree with the study conducted by Ebeed et al. ${ }^{(12)}$ as we had one case in whichinadequate breath-holding leading to false-negative interpretation by the viewer. We also had one false positive case due to misinterpretation of an irregularthick perilesional transient hyperemia by the viewer which resolved at the consequent study.

As tumour cells undergo necrosis, they present less of an impediment to the motion of water molecules. This results in a relative decrease in diffusion restriction and an increase in $\mathrm{ADC}$ value.

Taouli and Koh. ${ }^{(13)}$ agree with the following study conducted by Kamel et al. ${ }^{(8)}$, Vossen et al. ${ }^{(14)}$, Buijs et al. ${ }^{(2)}$ and Liapi et al. (5) that tumour necrosis corresponded to higher ADC values compared with viable tumour

In the current study, we agree with the conclusion of Goshima et al. ${ }^{(15)}$ that DWMRI was not found to be a reliable predictor of local HCC recurrence after TACE as compared with gadolinium-enhanced MR imaging and the difference between ADC variables between the viable and non-viable groups were statistically significant (P-value $<0.001)$. The best cut off that maximizes sensitivity and specificity is $\leq 1.22$. At this $\mathrm{ADC}$ value, the sensitivity is $83.3 \%$, and specificity is $71.4 \%$,

In the current study, we also found that diffusion MRI increased the sensitivity of local HCC detection and despite the decreased sensitivity yet there is a slight improvement of the overall accuracy.

We were convinced by the interpretation introduced by $\mathrm{Yu}$ et al. ${ }^{(16)}$ that the increase in false-positive findings originated from perilesional parenchymal insult lead to hypercellularity intermingled with a fibrotic component in the inflammatory granulation tissue which could restrict water diffusion, resulting in sustaining hyperintensity on DWI.

\section{Conclusion:}

The current study concludes that dynamic MRI is still superior than Diffusion-weighted MR imaging with high specificity and remain the standard follow up technique in well cooperative patient yet DWI is adding more to the overall sensitivity when combined with it and may help in condition of difficulty breathholding.

\section{REFERENCES}

1. Wang Z, Lin M, Lesage D, et al.: Threedimensional evaluation of lipiodol retention in HCC after chemoembolization: a quantitative comparison between $\mathrm{CBCT}$ and MDCT. Acad Radiol 2014; 21(3):393-39.

2. Buijs M, Vossen JA, Hong K,et al.: Chemoembolization of hepatic metastases from ocular melanoma: assessment of response with contrast-enhanced and diffusion-weighted MRI.

3. Lim HS, Jeong $\mathrm{Y}$ Y, Kang HK, et al.: Imaging features of Hepatocellular Carcinoma After Transcatheter Arterial Chemoembolization and Radiofrequency Ablation. AJR 2006; 187: 341-349. 
4. Sorensen AG.Magnetic resonance as a cancer imaging biomarker. Clin Oncol 2006; 24 (20): 3274-81.

5. Liapi E ,Geschwind JF , Vossen JA, et al.: Functional MRI Evaluation of Tumor Response in Patients with Neuroendocrine Hepatic Metastasis Treated with Transcatheter Arterial Chemoembolization. AJR 2008; 190:67-73.

6. Yaghmai, V., Besa, C., Kim, E., Gatlin, J. L., Siddiqui, N. A., \&Taouli, B, et al.: Imaging assessment of hepatocellular carcinoma response to locoregional and systemic therapy. American Journal of Roentgenology,2013, 201(1), 80-96.

7. Stuart K.: Chemoembolization in the Management of Liver Tumours. Oncologist. 2003; 8(5):425-37.

8. Kamel I, Bluemke D, Eng J, et al.: The Role of Functional MR Imaging in the Assessment of Tumor Response after Chemoembolization in Patients with Hepatocellular Carcinoma. J VascInterv Radiol 2006; 17:505-512.

9. Kim S, Mannelli L, Hajdu Ch, et al.: Hepatocellular carcinoma: assessment of response to transarterial chemoembolization with image subtraction. J Magn Reson Imaging 2010; 31: 348-355.

10. Braga L, Altun E, Armao D, and Semelka R $\mathrm{C}$, et al.: The liver in Semelka R C (eds). Abdominal-Pelvic MRI, John Wiley \& Sons Ltd., 4th edition. 2016; P39- P393.
11. Özkavukcu, E; Haliloglu N; Erden A et al.: Post-treatment MRI findings of hepatocellular carcinoma. Diagn Interv Radiol, 2009, 15.2: 111-20.

12. Ebeed A Ebraheem, Romeih M A, Refat M $\mathrm{M}$, Yossef $\mathrm{M} \mathrm{H}$, et al.: Role of dynamic contrast-enhanced and diffusion-weighted MRI in evaluation of hepatocellular carcinoma after chemoembolization The Egyptian Journal of Radiology and Nuclear Medicine 48 (2017) 807-815

13. Taouli B and Koh DM:Diffusion-weighted MR Imaging of the Liver, Radiology 2010; 254: 47-66.

14. Vossen JA, Buijs M, Kamel IR, et al.:Assessment of tumor response on MR imaging after locoregional therapy. Tech VascIntervRadiol 2006; 9:125-132.

15. Goshima S, Kanematsu M, Kondo H, et al.: Diffusion-weighted imaging of the liver: optimizing $b$ value for the detection and characterization of benign and malignant hepatic lesions. J Magn Reson Imaging 2008; 28(3):691-7.

16. Yu J, Kim J, Chung J, et al.:Added Value of Diffusion-Weighted Imaging in the MRI Assessment of Perilesional Tumor Recurrence after Chemoembolization of Hepatocellular Carcinomas. Journal OF Magnetic Resonance Imaging 2009; 30:153-160. 


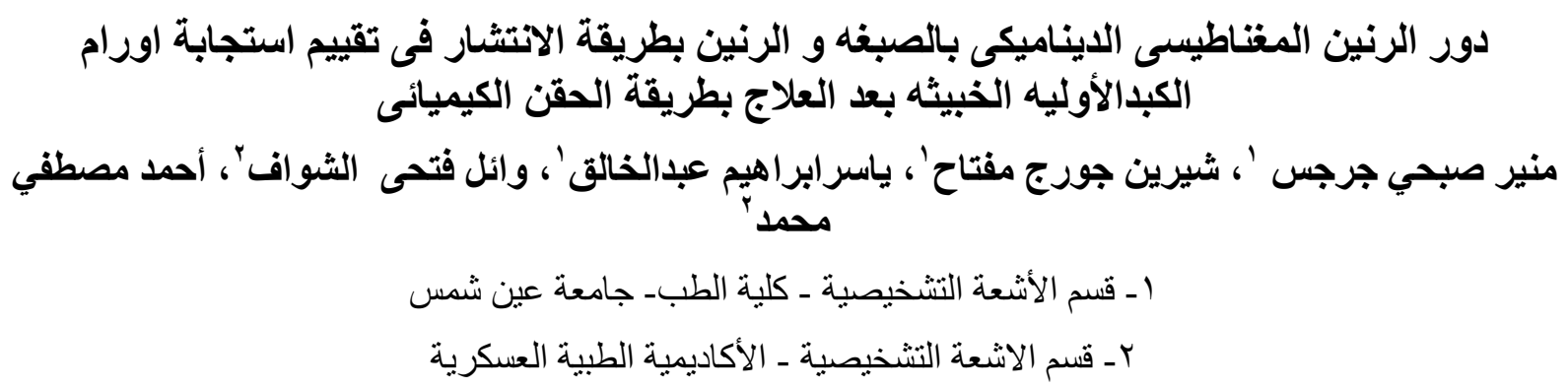

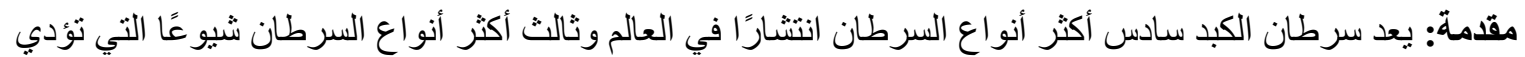

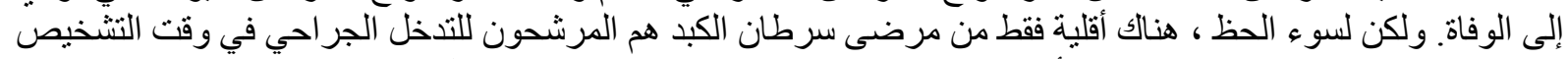

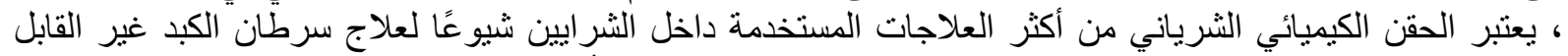

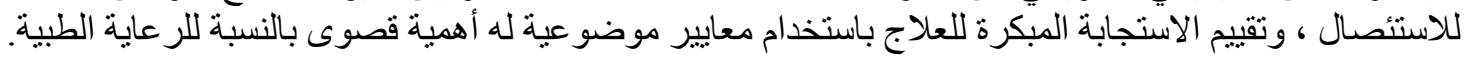

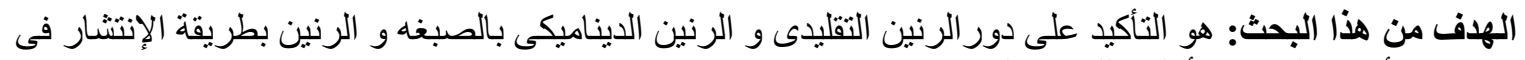

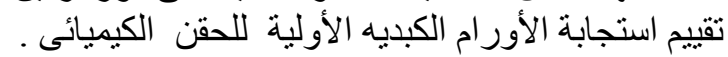

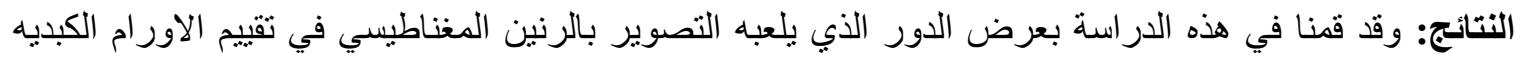

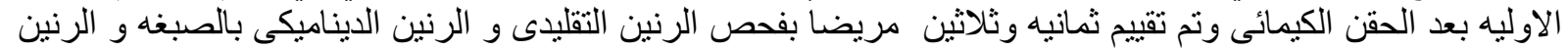
بطريقة الانتشار و تمت در اسة نتائج هذه الحالات.

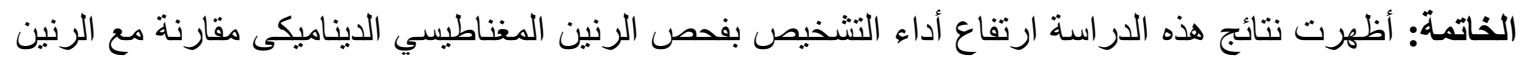

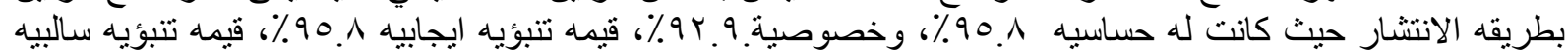

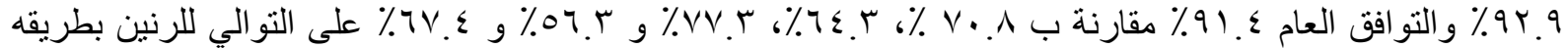

